\title{
Un etnomusicólogo en «escucha» (kûrûchobea) con los emberá chamí: una performance chamánica en los Andes
}

\author{
Juan Carlos Molano Zuluaga \\ (iD) https://orcid.org/0000-0003-1788-9873 \\ Universidade Federal do Rio Grande do Sul, Brasil \\ Juanmolano21@hotmail.com
}

RESUMEN

Este artículo se enmarca desde una provocación de carácter teóricometodológico basado en la experiencia intercultural de audición que tuvo en la década de 1970 el antropólogo colombiano Luis Guillermo Vasco con las prácticas chamánicas de los emberá chamí. Este lineamiento permite determinar algunos cuestionamientos con respecto a la posicionalidad en campo de este antropólogo en lo que respecta a la escucha, como un oído que atendió y tradujo ciertas significaciones auditivas a ciertos parámetros culturales. Ahora bien, estas interpretaciones etnomusicológicas, están dadas a partir de estas directrices y confrontadas por la inserción y participación en campo en un evento de "toma» o ingesta de ayahuasca en el resguardo indígena emberá chamí de San Lorenzo. La discusión ahonda en la imaginación y conceptualización indígena sobre el sonido, que merece especial atención en la etnomusicologíal antropología auditiva y que podría problematizar algunas teorías, como por ejemplo el perspectivismo amerindio. Sin embargo, la intención acá y basada en esa concedida intersubjetividad en el territorio indigena, es provocar consideraciones en torno a un concepto ontológico que llamaría "percepcionismo sónico», que es atribuido, como su nombre lo indica, a las percepciones de un determinado paisaje sonoro y su intangibilidad de territorio(s) y que podría complementar la teoría perspectivista que cimenta sus 
interpretaciones en una corporalidad tangible definida solo por su visibilidad.

Palabras clave: etnomusicología, semiótica musical, indígenas, emberá chamí.

\section{A non-indigenous ethnomusicologist in «listen» with the Emberá Chamí}

ABSTRACT

This article is a proposal from a framework of theoretical-methodological nature based on the intercultural experience of hearing that the Colombian anthropologist Luis Guillermo Vasco had in the 70's with the shamanic practices of the Emberá Chami. This guideline, gives an insight into determining some questionings with respect to the fieldwork undertaken by this anthropologist concerning the listening; as an ear that cared and translated certain hearing significances to certain cultural settings. Meanwhile, these ethnomusicological interpretations are given from these guidelines and confronted by my insertion and participation in the fieldwork in an event of «toma» or intake of ayahuasca in the Emberá Chami indigenous reservation from San Lorenzo.

The debate deepens in the indigenous conceptualization and imagination about the sound that deserves special attention in the auditory anthropology/ethnomusicology and that could confirm or contradict some theories as in the case of the Amerindian perspectivism. However, the intention here and based on that given intersubjectivity in the indigenous territory is to elicit considerations around an anthological concept that I would call "sonic perceptionism》, which is assigned; as its name suggests, by the perceptions within a certain soundscape and its intangibility of territory(ies) and that could complement the perspectivist theory that supports its interpretations into a tangible corporality defined only by its visibility.

Keywords: Ethnomusicology, musical semiotics, indigenous. 


\section{INTRODUCCIÓN}

Las distintas maneras en que las sociedades se relacionan entre sí a través del sentido del oído han proporcionado importantes elementos de estudio para antropólogos, sociólogos, filósofos, musicólogos y etnomusicólogos, convirtiéndose esto en un factor metodológico determinante para aproximar una realidad cultural. La antropología de los sentidos ha dado cuenta de esto en sus estudios al cuerpo y la percepción sensorial. Sin embargo, pocos son los que realmente se han acercado a algo más que solamente datos etnográficos, connotando en sus textos, interesantes posiciones y cuestiones teórico-metodológicas (Stoller, 1989; Taussig, 1993; Seremetakis, 1994; Howes, 2003).

Desde la década de 1980, vienen vislumbrándose interesantes posiciones frente a la percepción auditiva (la escucha) como un vínculo importante de interpretaciones sensoriales. Por ejemplo, el antropólogo (Feld, 1996) replanteó posiciones metodológicas por una antropología/etnomusicología del sonido, donde motivó una mirada hacia la percepción, significación e interacción humana con los sonidos de los entornos. Asimismo, acuñó el concepto de «acustemología» para hablar de una «epistemología acústica» que relaciona, la escucha como un conocer a través de lo audible. Por otro lado, el antropólogo James Clifford, menciona perspectivas que marcaron un rumbo metodológico etnográfico para investigadores, cuando postuló el cuestionamiento, «pero ¿qué de la oreja [oído] etnográfica[o]?» (Clifford y Marcus, 1986, p. 12).

Tanto Feld, desde la antropología/etnomusicología, como Clifford, desde la antropología, han localizado un camino, para pensar o (re)pensar la acción de la escucha-conocimiento. Estos autores, desde sus denotadas posiciones epistemológicas, consideran que el estar a la escucha, nos coloca en una posición distinta frente al mundo, permitiendo apreciar aspectos del entorno y de una propia subjetividad, que suelen ser ignorados cuando la bloqueamos o no la «comprendemos». 
En otras palabras, conceden un componente insospechado para la acción perceptiva del «oír», «escuchar», no a través de fórmulas y mecanismos ya conocidos y aprendidos por una trayectoria de vida, sino a través de la apertura hacia esa voz de alteridad. Como lo inspira Feld cuando denota que escuchar al otro ( $\mathrm{u}$ entorno natural o sobrenatural) implica, si se hace con atención, escuchar una escucha diferente, postulado que es enunciado por otros trabajos como Erlmann (2004), Oliveros (2005), Nancy (2007), Lenkersdorf (2008), Voegelin (2014) y Ochoa Gautier (2015).

Desde esta perspectiva, este texto, da cuenta de la importancia de la escucha y el sonido para la sociedad indígena emberá chamí y sus propósitos perceptivos de comunicación e intervención sonoro-espacial, para establecer y mediar ciertos beneficios comunitarios. Esta sociedad indígena andina estimula un complejo proceso de escucha de todo un pluriverso sonoro, para conceder vínculos territoriales y ancestrales con fines curativos. Es decir, la escucha, pero más precisamente la «percepción sonora», establecida como un aditamento significativo para alcanzar relaciones o vínculos con las distintas fuerzas que componen el mundo natural y sobrenatural (la naturaleza, humanos, no-humanos, animales, etcétera). Finalmente, el texto dimensiona todo un camino metodológico otorgado para significar e interpretar ciertos postulados epistemológicos, norteados por una inevitable posicionalidad del yo-investigador (Abu-Lughod, 1991), como sujeto que media, es agente y también «escucha» en ese espacio intercultural.

\section{EN ESCUCHA CON LOS EMBERÁ CHAMÍ}

Los emberá, êbêra o ẽpẽrá, también llamados chocó, son un pueblo amerindio que habita algunas zonas del litoral pacífico y zonas adyacentes de Colombia, el nororiente de Panamá y el noroccidente del Ecuador. Los que habitan los Andes colombianos se conocen como emberá chamí o personas de la montaña, que por distintos motivos sociopolíticos han seguido un patrón de poblamiento disperso entre las cordilleras central y occidental. Así, como sucede con la lógica predominante con otras sociedades amerindias, los emberá chamí vislumbran, dentro de sus filosofías y ontologías indígenas, múltiples percepciones sobre el sonido que se constituyen como un dispositivo importante para acceder a nuevas formas de conocimiento y de ser/estar indígena. 
Una mañana en San Lorenzo ${ }^{1}$, mientras doña Martha molía maíz en la cocina con sus hijas, me manifestó:

$[\ldots]$ ¡Darío! ${ }^{2} \ldots$ a él lo llaman mucho para hacer trabajos en Riosucio ${ }^{3},[\ldots]$ yo lo acompaño la mayoría de veces... la última vez, fue bien difícil, porque a mí se me pegó un espíritu... ese día, estábamos en círculo... pues, Darío estaba en el trabajo [de curación-sanación], cuando vi que del zapato de la señora, que se le estaba haciendo el trabajo, se le salió una cucaracha... me dio como susto, porque esa cucaracha se le empezó a subir a Darío por el pantalón... ipor dentro del pantalón!... yo decía, ¡Darío! ¡Darío! ¡Darío! ¡Cada vez más fuerte!... pero Darío no podía parar, con los cantos y el trabajo de él... porque es peligroso... por los espíritus... entonces... caí enferma y me desmayé.

Las prácticas chamánicas se basan en una profunda interrelación entre la persona y el mundo natural circundante que lo rodea, social y sobrenatural. Así lo referencia Vasco (1985) al acotar que, en el caso de los emberá, diferentes autores han considerado que la enfermedad es producida por la acción de algún espíritu (jai) maligno que los castiga. Así, la salud depende estrechamente de estos vínculos, con los factores ecológicos y los seres que la habitan, y como me indicaron algunos interlocutores mientras realizaba el trabajo de campo: esos espíritus son los dueños o guardianes que determinan las formas en que se deben adquirir las relaciones entre los humanos, los no-humanos, los animales y la naturaleza. Cuando doña Martha expresa: «[...] entonces... caí enferma y me desmayé», es producto de la posesión (bana) de un espíritu o jai maligno en el cuerpo de ella.

Vasco $(1975,1985)$ señala que los indígenas emberá designan con el nombre de jaibaná a la persona que cura o sana con su canto y sus bastones jai ${ }^{4}$, empleando para sí el conocimiento ancestral y la utilización de las plantas medicinales. En este sentido, los jaibanás usan sus cantos (kima kima) y bastones para establecer

1 San Lorenzo es un territorio indígena emberá chamí, localizado en el noroccidente del departamento de Caldas en la cordillera occidental colombiana. Es un territorio con una población aproximada de 12000 indígenas, distribuidos en veintiún comunidades.

2 Don Darío es un músico y médico tradicional emberá que vive en San Lorenzo. Su conocimiento en etnobotánica la aprendió precisamente de algunos chamanes de la Amazonía colombiana y por lo mismo, le ha otorgado un grado de referencialidad en sus prácticas chamánicas o de medicina tradicional, tanto dentro como fuera del territorio indígena.

3 Municipio de Caldas con una masiva movilización indígena por su proximidad al territorio indígena de San Lorenzo.

4 Son artefactos o «varas» - bastones - de madera tallados, que son ya poco usados por los emberá y constituían parte tacita e integral de la presencia del chamán o jaibaná para sus propósitos de curación. 
ese puente de conexión entre esos otros mundos habitualmente separados, como el de los espíritus o el de los muertos, precisamente, para curar cuerpos enfermos $^{5}$. Ahora bien, para ejemplificar estas líneas, Vasco (1985) hace mención en su trabajo a un jaibaná «arrepentido» llamado Clemente, quien había destruido sus bastones por la presión de la administración pública y de la iglesia católica. Este hecho marcó e irrumpió de una u otra forma la concepción de los cantos y artefactos sonoros en las prácticas chamánicas emberá. Es decir, el destruir los bastones, fue un acto difícil y peligroso, puesto que se dice que es como matar a su dueño. Por tanto, Clemente tuvo que superar este suceso y continuar su vida sin bastones. Sin embargo, por una necesidad de curar dieciséis años después a un nieto suyo quien estaba gravemente enfermo, Clemente decidió cantar jai sin sus bastones y logró curar al niño.

A raíz de estos acontecimientos históricos, es pertinente plantear algunos interrogantes con respecto a las actuales prácticas chamánicas o de medicina tradicional en estos territorios indígenas. Entonces, ¿cómo está resignificado hoy el jaibanismo? ¿Qué escuchan y cómo escuchan los emberá en las prácticas chamánicas o de medicina tradicional? Estos dos interrogantes podrán ser pretenciosos, especialmente el segundo, debido a su carácter «aprehensivo» por el investigador de estas ontologías sonoras. Sin embargo, es importante señalar que constituyen en su construcción semántica, un esbozo para delinear efectiva y afectivamente estos caminos y así aproximar interpretaciones en torno a esas epistemologías nativas.

El primer interrogante postula una interpretación histórica en cuanto el papel del jaibaná y la resignificación de sus prácticas en los territorios indígenas emberá, y el segundo interrogante, constituye el método de investigación, que se adoptó para solventar estas interpretaciones etnomusicológicas. Es decir, el dejarme afectar por esas lógicas nativas en campo implicó la ingesta de la planta de ayahuasca con el fin de obtener mejores posibilidades para intentar comprender esas filosofías y ontologías sonoras, esos mundos audibles e inaudibles del cual está conformada la cosmo-sónica ${ }^{6}$ emberá chamí.

5 En mi trabajo de campo, evidencié que la palabra jaibana está ya poco usada en estos territorios indígenas, debido a las confrontaciones y presiones de décadas pasadas por ciertas políticas misioneras, justamente, por la realización de estas prácticas chamánicas en los territorios indígenas.

6 Stein (2009) elaboró un concepto-síntesis con la sociedad indígena Mbyá-Guarani sobre las fuertes imbricaciones entre las dimensiones sociocosmológica y sonora que denominó de «cosmo-sónica». Este término apunta "para la centralidad del ámbito sonoro en el modo de ser Mbyá, subrayo, desde ya, que la cosmo-sónica más precisamente debería ser acuñada como 


\section{DEVELANDO UN MÉTODO ETNOGRÁFICO}

Evidenciar ciertos caminos de interpretaciones sónicas, relacionadas y pensadas desde un desempeño netamente de carácter metodológico, siempre fue motivo de preocupación desde el primer momento que entré a campo con los emberá chamí. En este sentido, como investigador puedo discernir sobre un interrogante que se basa en la posición de «escucha» de un músico con una «aparente» formación dentro del canon estético sonoro-musical occidental e investigador no-indígena, para con ciertas prácticas performativas sonoras indígenas. Entonces, ¿cómo nuestro oído debe estar "condicionado» en campo, sabiendo que la mayoría de nuestras interpretaciones giran alrededor de este sentido, aun mas, considerando que nuestro oído tiene toda una vida de experiencias auditivas previas o anteriores que van a entrar también a relacionar estímulos perceptivos junto con las vividas en campo?

Seeger $(2015$, p. 30) trae una reflexión desde su experiencia etnográfica con los conceptos de «oído etnográfico» y «oído indígena». Este autor considera elementos importantes del oído etnográfico, aludiendo a una suerte de sensibilidad particular hacia los mundos sonoros de grupos étnicos, y el oído indígena como un elemento exclusivo del cual el etnomusicólogo o etnólogo debe intentar comprender. En otras palabras, el oído etnográfico no es lo mismo que el «oído de un etnógrafo» porque el primero, es producto de ese encuentro intersubjetivo entre el investigador, colaboradores e interlocutores, quienes conjuntamente focalizan y discuten el sonido, mientras que el segundo, es un tipo de «condicionamiento sónico» que se deriva de toda esa trayectoria auditiva en la vida del investigador, que por supuesto, no se obvia cuando entra a campo.

Entre los emberá, Vasco $(1975 ; 1985)$ fue el pionero en considerar fuentes en lo que respecta a la importancia que tiene el sonido para esta etnia. Este antropólogo colombiano denotó, en la década de 1970, un particular interés en los sonidos $(j t k u a)^{7}$ y el canto de esta sociedad, enfatizado, como ya se había mencionado, en el papel del jaibaná. A pesar de que Vasco, se encontraba espacialmente posicionado como antropólogo en contextos netamente coloniales, es decir, una década permeada por distinciones de poder en el cual las apreciaciones por algunos de

\footnotetext{
'sociocosmo-sónica' por envolver el campo social, constituido también por la musicalidad, entre tanto, por creer que la sonoridad 'cosmo-sónica' es más accesible a los oídos y a los diálogos, preferí omitir lo 'socio' y producir una metonimia, sugiriendo que en el 'cosmo' se incluyen las relaciones humanas y extra humanas» (p. 24, traducción propia).

7 Palabra emberá para designar sonido.
} 
sus colegas, y especialmente misioneros, para con estas identidades indígenas y sus prácticas eran de «primitivas», "sacrílegas» y «prerracionales»; es preciso indicar que su constructo y pensamiento en cuanto estas interpretaciones sónicas, probablemente desbordaron otros intereses.

No obstante, tratar de inferir un interrogante, podría solventar directrices en cuanto el oído espacial y temporal de este antropólogo en cuestión, y así, poder relacionar actualmente estímulos perceptivos con las prácticas chamánicas de estos actores sociales. Entonces, ¿qué escuchó y cómo escuchó Vasco los sonidos del jaibaná Clemente en su trabajo de campo, considerando los condicionamientos de orden estético, científico e ideológico de la época?

Para responder esta pregunta, García (2015), en su trabajo, señala el siguiente enunciado epistemológico de Boaventura de Sousa Santos (2009): «Cada método es un lenguaje y la realidad responde en la lengua en que es preguntada» (p. 49). Este postulado, ofreció un camino para García, al interpretar las dimensiones e impresiones en cuanto a las condiciones de audición que despierta ese encuentro con la otredad. Es decir, que, según estas interpretaciones, toda manifestación sonora es percibida desde las disposiciones auditivas del sujeto que escucha. Entonces, ¿quién escuchó al jaibaná Clemente y sus prácticas sonoras? ¿Fue el oído de Vasco? ¿Fue el oído de los lineamientos científicos e ideológicos de la época? ¿O fue el oído colonizador de la época?

Aunque Vasco en su trabajo no fue más allá de una descripción de la práctica chamánica del jaibaná y su canto, es decir, no dimensionó interpretaciones etnomusicológicas, sí deja entrever en su escrita etnográfica que él escuchó al jaibaná en su práctica de curación-sanación en ese territorio indígena, lo que conduce a esbozar su probable experiencia intercultural de escucha, como un oído que atendió y tradujo ciertas significaciones auditivas a ciertos parámetros culturales ${ }^{8}$.

Por lo tanto, otorgar aquí un beneficio metodológico en este sentido es relevante, conferido desde una provisionalidad inherente a mi ser en relación con los mundos sonoros que me rodean. Entonces, una «audición insurgente» $\mathrm{o}$ «audición desobediente», como lo menciona García (2015), podría entregar caminos más loables de escucha. Es decir, la audición insurgente o audición desobediente que

8 En esta época el consumo de alucinógenos por estas identidades indígenas, especialmente jaibanás para intervenir prácticas chamánicas, estaba relacionado con la toma de bebidas fuertes y embriagantes como la chicha de maíz, chontaduro y panela y el aguardiente; este último adquirido en las ciudades. Sin embargo, en ocasiones, a las bebidas eran adicionados otros alucinógenos como la datura y el banisteriopsis caapi «pildé», también conocido como ayahuasca o yagé. 
se levanta contra la autoridad canónica sónica contemporánea, que se subleva contra esos poderes culturales, especialmente estilísticos y que podrían hacer emerger otro tipo de audición.

En este sentido, el recurso metodológico para afrontar estas interpretaciones etnomusicológicas se da precisamente a partir de estas consideraciones en campo y que conducen a determinar la posición y «condición de escucha» del investigador. Una audición insurgente o audición desobediente también otorgada en este sentido, a lo que podría determinar por otra condición de percepción sónica, atribuida inclusive por la alteración sensitiva de la visión, el olfato y por supuesto la escucha.

\section{EN «ESCUCHA» (KÛRÛCHOBEA) CON LOS EMBERÁ CHAMÍ: SER AFECTADO}

En uno de esos días de trabajo de parcela ${ }^{9}$ en la finca de la familia ${ }^{10}$, don Darío me expresa muy animado, mientras escoge la leña con la que va para prender el fogón del almuerzo: «En los próximos días viene el taita Emiro, aquí a la finca, a darnos medicina». El taita ${ }^{11}$ Emiro es un chamán de aproximadamente setenta años, no-indígena, del alto Putumayo de la Amazonia colombiana, que constantemente llega a estos territorios indígenas andinos, especialmente a San Lorenzo, para realizar prácticas de ingesta de ayahuasca. El taita, como él mismo lo expresó días después de su llegada, se define como un «colono», que aprendió las tradiciones y costumbre ingas ${ }^{12}$ y que llega a estos territorios indígenas precisamente para curar y sanar enfermos.

9 Es una pequeña porción de terreno que tiene cada familia indígena para su uso agrícola, entregada por el gobierno indígena.

10 Es la familia de músicos indígenas emberá que me acogió desde los primeros días de mi trabajo de campo en San Lorenzo, personas con las que compartí y aprendí las distintas labores agrícolas y caseras. La familia está conformada por don Darío, su compañera — esposa— doña Martha, sus hijos (Nodi, Esmo, Aura, Nelsa, Samabe, Jenara, Nepima), su nieta Nejuesta y su yerno Fredy.

11 Es el nombre con el que se denomina al chamán en estos territorios indígenas y en la Amazonia colombiana. Los conocimientos de los taitas están articulados al uso y administración del «yagé» o ayahuasca. Ahora bien, el «yagé» o ayahuasca es un bejuco selvático que se da en el piedemonte amazónico de Colombia, Perú y Ecuador. Las sociedades indígenas andinas en Colombia en las últimas décadas han intensificado el consumo de este alucinógeno en sus ceremonias y rituales.

12 El pueblo indígena inga o ingano es un grupo quechua que habita en el Putumayo (suroccidente colombiano), Amazonia colombiana. 
Los días siguientes en la finca fueron de intensos preparativos, donde me inmiscuí junto con la familia en tareas para con el evento de la ingesta o «toma» ${ }^{13}$ que precisamente sería en el espacio sagrado de su parcela ${ }^{14}$. Algunas actividades, tales como: conseguir algunas carpas y plásticos, recoger abundante leña, adecuar el sitio sagrado y preparar algunos alimentos, fueron las principales indicaciones que don Darío manifestó a su familia para no pasar contratiempos el día del evento.

En las vísperas de la «toma», la incertidumbre y ansiedad estuvieron siempre latentes, debido a mi posición identitaria como persona no-indígena e investigador. Es decir, quería presenciar el evento, deseoso sobre todo de «escuchar» las dimensiones sónicas dadas por las performances y observar la sociabilidad de las personas en condiciones de ingesta, pero no tenía certeza alguna sobre si participar o no de la «toma».

Durante toda la convivencia con la familia indígena, siempre estuve dispuesto a dejarme afectar por las situaciones y los momentos compartidos; contemplando así, mejores posibilidades en cuanto al privilegio de vivir esas filosofías y ontologías indígenas. Sin embargo, en esta ocasión, mis dudas estaban latentes, precisamente porque no encontraba respuestas claras que me brindaran confianza para participar activamente de esta ceremonia (Ukaraba kirú). No obstante, días después don Darío me otorgó algún beneficio de confianza, al manifestarme que la decisión de participar de la ingesta no estaba en mí, sino en la misma planta que me elegiría para ser sanado.

Estas palabras abrieron otro portal de interrogantes de los que en ninguna otra situación habría podido preguntar, o ni siquiera pensar. Para discurrir en esta disyuntiva, Favret-Saada (2005) muestra precisamente esa divergencia en el tratamiento metodológico. Es decir, esta autora, en su trabajo de campo en el Bocage en el noroeste francés, evidencia el postulado de si es relevante y preciso para estudiar la brujería, no solo observarla, sino, además, dejarse afectar por sus lógicas. En este sentido, coincido con estos argumentos y pensamientos al asumir y enfrentar una posición en campo. En palabras de la autora:

[...] en el comienzo no paré de oscilar entre esos dos obstáculos: si yo «participase» el trabajo de campo se tornaría una aventura personal, esto es, lo contrario de un trabajo, pero si tentase «observar» quiere decir mantenerme

13 Algunas de las palabras con las que los indígenas identifican la planta de la ayahuasca son: yagé, el remedio, la medicina, el elemental.

14 El sitio es denominado sagrado porque es el espacio que interviene la familia e indígenas del territorio para congregarse a realizar las prácticas de medicina tradicional o prácticas chamánicas. 
a la distancia, no hallaría nada para «observar» [en mi caso nada para «escuchar»] (Favret-Saada 2005, p. 157, traducción propia).

El día de la ceremonia todo estaba preparado, y al caer la noche, el lugar sagrado adquirió un aditamento mágico. Alrededor, en esa inmensa naturaleza desplegada por infinidad de sonidos que emergen principalmente de los pronunciados vientos característicos de las tierras altas, me encuentro con personas invitadas no-indígenas y personas indígenas del resguardo, que en esta ocasión llegaron a la finca. No podría detallar muy bien el número de personas en el espacio, pero aproximadamente entre hombres, mujeres y niños sumaban unas cincuenta. Así pues, empieza a cantar el taita, con la cabeza inclinada moviendo su waira ${ }^{15}$, detrás de una mesa con ofrendas y flores. Por largos minutos, emite unos cantos onomatopéyicos con un ritmo lento y cadencioso en un lenguaje incomprensible que salen precisamente por los largos sorbos de aguardiente preparado ${ }^{16}$ que toma y espira hacia arriba para expulsarlo.

Seguidamente, abruptamente interrumpe su canto y expresa a los asistentes:

[...] dar las gracias a doña Martha y al taitica Darío por este sitio tan mágico que tienen acá, [...] cada uno, a lo que vengamos, venir con un propósito para el espíritu del yagé... por enfermedades, por salud, etcétera [...], los elementales están a toda hora con nosotros, $a h o !^{17}$

Por otro lado, don Darío quien se encuentra al lado del taita, como un colaborador asiduo de sus prácticas chamánicas en estas tierras altas, también interviene y dice:

[...] nos encontramos hoy aquí alrededor del fuego y la intención es que cada uno traiga un propósito y lleve pues como ese objetivo, como para que se van a tomar la medicina [...] esta es una fiesta de reflexión, de curación, esto no es como lo que se decía antes... que esto es satánico, ¡no! Esto es curación, donde la medicina va a limpiarles... ese elemental... ese gran espíritu se va a comunicar con ustedes.

15 Es un artefacto chamánico hecho de hojas secas.

16 Es un destilado de caña con algunas plantas medicinales preparado por los indígenas. Es llamado comúnmente pelaguache.

17 Es un vocablo de la lengua lakota, resignificado por estas sociedades indígenas andinas. Es frecuentemente usado en conversaciones en este tipo de celebraciones rituales con la ayahuasca y significa: por todas «mis relaciones», o lo que es lo mismo, «todos estamos relacionados». 
Después de estas intervenciones, el taita empieza a interpretar una armónica ${ }^{18}$, que acompaña con unos fuertes zapateos, en un ritmo constante, sin dejar de lado su waira que tiene en su mano derecha y balancea sobre el yagé. Acto seguido, el taita cuidadosamente prepara la bebida, vertiendo su liquido en un pequeño totumo que ofrece a los asistentes. A partir de este instante, no sé si todo empezó a transcurrir más de prisa o más lento, faltaron solo unos segundos para perder la noción completa de tiempo. El estar afectado, creo que fue algo verdaderamente osado, donde, el «escuchar» los sonidos de la naturaleza en la noche, los murmullos de las personas y los cantos del taita me concedieron otra forma de ser/ estar en el espacio. Como menciona Viveiros de Castro (1992), ser afectado por esas filosofías indígenas cambia la visión de cuerpo en esa mirada ontológica.

Ahora, mi disposición está centrada en escuchar o más bien «escuchar» ${ }^{19}$ toda la dimensión sónica que se percibe en el entorno (ankoré) ${ }^{20}$. Los sonidos de la naturaleza se tornan más perceptibles y mi oído tiende a querer manifestarse distinto al «escuchar» mayores referencias sónicas. Esta aparente «elongación» sensitiva de mi sentido procura accesos a discriminar más rápidamente los sonidos. El paisaje sonoro hace eco de los cantos del taita, que esta vez van acompañados de unos fuertes acordes que ejecuta en una guitarra, pronunciando frases en español, que al parecer improvisa a medida que va recorriendo el entorno. Dentro de las frases que alcanzo a percibir incluye los nombres de algunas de las personas presentes, al parecer como si fueran plegarias de sanación. Al respecto, Vasco (1985), menciona que al jai no hay que entenderlo como espíritu, sino como la esencia misma de las cosas o una energía. No es por acaso que esas manifestaciones sonoras se magnifican, elevan y transforman formando parte integral de una misma esencia.

Dentro de esa variedad sónica que se despliega en este lugar sagrado, alcanzo a percibir a lo lejos los gritos «desesperados» de una de las personas. Al percatarme, observo que es uno de los indígenas, que se encuentra postrado en el suelo, al parecer en una especie de trance severo por la ingesta, «la rasca» ${ }^{21}$. Describir la sensación al «ver» y «escuchar» este indígena no es lo más preciso acá, sin

18 Este instrumento musical de soplo metálico junto con la guitarra es frecuentemente usado por los chamanes y médicos tradicionales de los andes, jugando un papel importante en la musicalidad de la ceremonia donde se interpreta en solitario o en conjunto. «Escuchar» entre comillas, la uso para designar la acción de oír o escuchar afectado sensitivamente por la planta de la ayahuasca.

20 Palabra emberá para designar mundo-cosmos.

21 Categoría nativa para describir un estado en la «toma» de ayahuasca. Es decir, cuando se limpia el cuerpo de «energías negativas» llega «la rasca». Al desintoxicar el cuerpo, se desintoxica 
embargo, puedo denotar que podría ser de angustia o miedo, probablemente, de creer que en segundos o minutos eso mismo me sucederá.

Indudablemente, fue a raíz de este acontecimiento que pude percibir más claramente lo que vagamente intentaba comprender aquel día en la cocina de dona Martha, cuando ella mencionaba su enfermedad, producto de la posesión de su cuerpo por un espíritu. Por consiguiente, toda la atención del taita y de don Darío para con el indígena enfermo fue relevante, en el sentido de intentar comprender toda la dimensión del papel del [jaibaná] o médico tradicional en función de estas prácticas de sanación. Días antes, don Darío ya me había mencionado que son los espíritus [o jai(s)] que deben estar bajo el control del taita para establecer nuevos contactos con otros espíritus [o jai(s)] para invitarlos a conversar. Estas palabras demuestran esa relación intensa con las alteridades, ese vínculo ancestral con ciertos espíritus, dados precisamente por las facultades de «escucha» del taita [o jaibaná].

\section{Figura 1. Espacio sagrado para la ceremonia (foto del autor)}

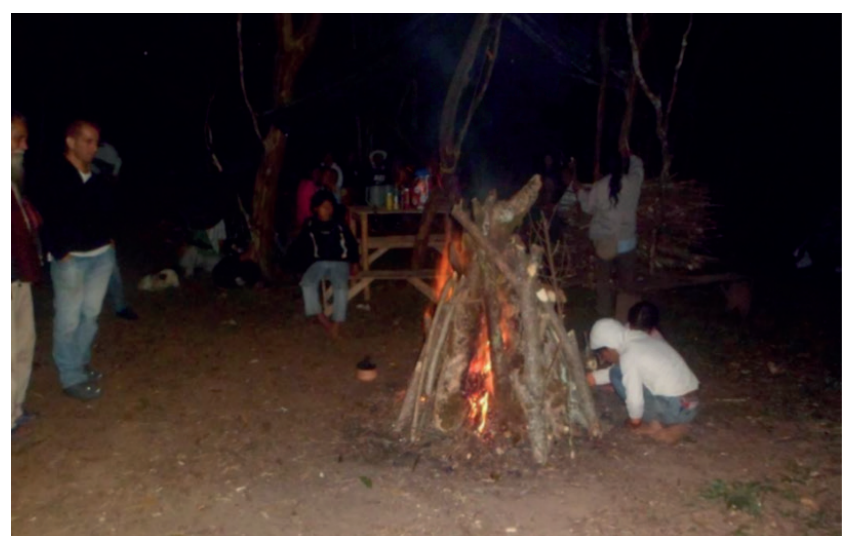

Foto: Juan Carlos Molano

El percepcionismo sónico: De los «puntos de vista» hacia los «puntos de escucha»

Para empezar este apartado, es importante recordar el interrogante planteado anteriormente, ¿Qué «escuchan» y cómo «escuchan» los sonidos en las prácticas

el alma. «La rasca», en términos nativos, «pega fuerte». Las personas gritan, se tiran al piso y patalean. Otras corren buscando un lugar para vomitar y buscar aire fresco. 
chamánicas de sanación-curación los emberá chamí? Este interrogante ha permitido explorar, a partir de los «condicionamientos sensitivos» de los que hago mención, una probable «perspectiva de audición» por estas identidades indígenas.

Para comprender esta postura, Viveiros de Castro define el perspectivismo amerindio como, «[...] especies de sujetos o personas, humanas y no-humanas, que lo aprehenden desde puntos de vista distintos» (2002, p. 348). Esta nueva forma amerindia le sugirió al autor la creación del término multinaturalismo, precisamente para diferenciarlo del multiculturalismo y que lo define como una «implicación mutua entre la unicidad de la naturaleza y la multiplicidad de las culturas, la primera garantizada por la universalidad objetiva de los cuerpos y de la sustancia, la segunda por la particularidad subjetiva de los espíritus y del significado» (Viveiros de Castro, 2002, p. 349).

Interpelar el concepto de «perspectiva» en el cual Viveiros de Castro fundamenta su teoría ayuda a ahondar en caminos netamente preponderantes debido a estas ontologías sonoras. Es decir, este concepto de «perspectiva» o «perspectivismo» que es atribuido desde unas nociones netamente visuales, conlleva a repensar la dimensionalidad del sonido en estas ontologías indígenas, asumiendo interrogantes en lo que respecta a que, si existen «puntos de vista» basados en la percepción del mundo, podrían coexistir ciertos «condicionamientos sónicos» que converjan en distintos «puntos de escucha» basados en la percepción del mundo.

Estas preocupaciones y consideraciones han sido planteadas también por Lewy (2015), en su trabajo etnográfico con los Pemón en Venezuela, donde sugiere cómo se deben comprender las relaciones entre las especies, si el «escuchar» está en el centro de las interpretaciones. Este autor, define el concepto de «sonorismo» complementario u opuesto al perspectivismo de Viveiros de Castro, contribuyendo a deducir una posible ontología de sonido por las identidades indígenas. En este sentido, es posible pensar un paradigma sonoro de comunicación transespecífica que se asemeja al perspectivismo amerindio.

Entonces, el paradigma según Lewy comprende:

1) los seres humanos escuchan a los seres humanos como seres humanos, a los animales como animales y/o seres humanos, y a los espíritus como seres humanos y/o animales; 2) los animales (presa) se escuchan a sí mismos como animales y/o seres humanos, a los seres humanos como seres humanos y/o animales; 3) los espíritus se escuchan a sí mismos como seres humanos y a los seres humanos como seres humanos y/o animales; 4) los animales depredadores escuchan a los seres humanos como seres humanos y a los animales como animales en su fisicalidad típica (2015, p. 96). 
Por tanto, Lewy provoca un foco de atención en estos mundos audibles e inaudibles de estas sociedades indígenas, derivadas desde los aportes del perspectivismo de Viveiros de Castro y ajustados para brindar significativas contribuciones teórico-metodológicos para la etnomusicología/antropología auditiva (Feld, 1982; 1996) y (Menezes Bastos, 1999a; 1999b; 2013).

No obstante, el otorgar mención al hecho de encontrar una «localización» para una interioridad o fisicalidad sobre estas posibles perspectivas de audición posibilita intentar comprender una interioridad o exterioridad, con base en lo audible e inaudible. Esto provoca cuestionamientos determinantes a otorgar caminos más loables de interpretaciones con estas ontologías indígenas. Así, como el perspectivismo amerindio cimienta sus interpretaciones en una corporalidad tangible definida por su visibilidad, donde la forma (visible) del cuerpo determina sus perspectivas (puntos de vista). Del mismo modo, se pueden provocar consideraciones en torno a lo que llamaría un «percepcionismo sónico», atribuido por las percepciones de un determinado paisaje sonoro y su intangibilidad de territorio(s); proyectada, por una condición de «escuchabilidad» ${ }^{22}$, donde el espectro sonoro determina su «percepcionismo», es decir, distintos «puntos de escucha».

Ahora bien, siguiendo estos acercamientos teóricos y reflexiones a la luz de la «escucha» del paisaje sonoro y las distintas performances sonoro-musicales en el evento de ingesta de ayahuasca, se podría discernir mejor sobre estas manifestaciones en torno al espectro sonoro y la recepción auditiva por estas identidades indígenas. Es decir, toda la intangibilidad de este espacio sagrado en la parcela de San Lorenzo, con relación a las practicas chamánicas, conduce a dirimir una total correspondencia entre el paisaje sonoro y las dimensiones sónicas, que se dan por los vínculos que el taita o chamán hace con los espíritus [o jai(s)] para propósitos curativos. En este sentido, tanto las performances sonoro-musicales, como las manifestaciones sonoras ecológicas circundantes del lugar, determinan la «localización» de esas probables «perspectivas de audición»; por tanto, la «escuchabilidad» se asume como una condición que procura visar estas identidades indígenas en estas prácticas chamánicas para propósitos curativos.

22 Este neologismo se entiende como el acto de captar unas realidades inaprensibles, es decir, una nueva escucha que rompa con los criterios rígidos y, a veces, fragmentarios de la escucha analítica. 
Figura 2. El percepcionismo sónico

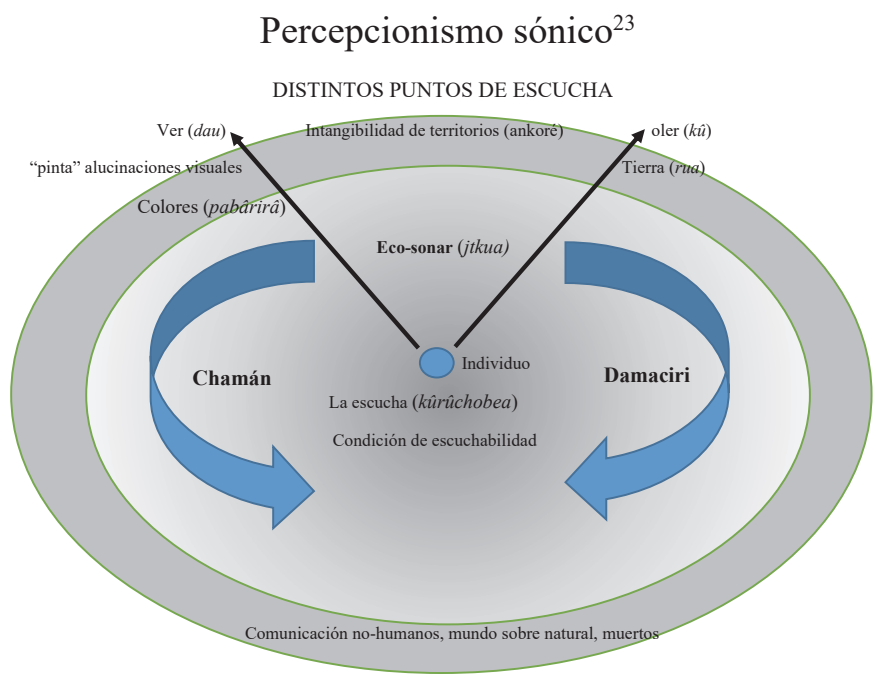

Elaborado por el autor y colaboradores indígenas.

\section{LA CURA}

Entrada la noche se manifiesta con más intensidad sónica este espacio sagrado. El entorno ecológico natural parece con más preponderancia hacer eco de cada una de esas manifestaciones sónicas circundantes, en este lugar paisajístico tropical de montaña. Los rezos, murmullos, respiraciones y agitaciones de cada una de estas personas presentes igualmente forman parte asidua de ese mundo sonoro en constante transformación que segundo a segundo se revela con más predominio. Es como si mágicamente la naturaleza ajustara o adaptara sus contornos para amplificar ciertos sonidos, que por más ínfimos o imperceptibles para ser discriminados al oído, más es su resonancia o reverberación en el espacio.

En este sentido, cobran relevancia las disposiciones cosmoespaciales para obtener esos beneficios curativos, en estas prácticas chamánicas, y adquieren sentido las palabras de don Darío cuando expresó «[...] Usted debe escuchar ese llamado, si es elegido para tomar la medicina [...] después en la toma, el taita y los sonidos le mostrarán los caminos. [...] usted, por eso debe escuchar muy

23 El gráfico es atribuido y esquematizado en campo a partir de discusiones con los emberá en torno a la escucha. El percepcionismo sónico se atribuye a un desarrollo sinestésico en torno a los sentidos del olfato, la visión y la escucha principalmente, producto de esa alteración sensitiva por la ingesta de ayahuasca. 
bien». Estas palabras, denotan esas posibles «perspectivas de audición» por estas identidades indígenas. Es decir, las disposiciones cosmosónicas que estas personas buscan con el sentido de la escucha es formar un vínculo con esa condición de «escuchabilidad». En otras palabras, la ingesta de ayahuasca es uno de los caminos que estas personas usan, para determinar de una manera casi fisiológica ese «condicionamiento sónico» que se dimensiona en esas otras formas de «ver» (dau), «oler» $(k \hat{u})$, pero sobre todo «escuchar» (kûrûchobea) emberá chamí.

Si bien el consumo de esta ingesta permite un condicionamiento sónico, también es importante reconocer la intención del chaman en esta práctica, al curar o sanar al indígena que se encuentra en un estado absorto o de «inconsciencia», que se refleja en su rostro y cuerpo. Según palabras del taita, esta situación obedece porque tiene un espíritu muy aferrado, producto del no reconocimiento de sus karmas o enfermedades por parte del indígena. En este sentido, Vasco (1985), reafirma esta particularidad cuando señala: «[...] un jai que se siente cómodo, se queda» (p. 18). Por lo tanto, mientras el taita toma posición con sus elementos chamánicos y artefactos sonoros para con su habitual performance de curación-sanación, don Darío, por su parte, inicia una performance musical con los integrantes de su grupo familiar Damaciri ${ }^{24}$ en un costado del espacio sagrado.

Las prácticas sonoro-musicales del grupo Damaciri se nutren de esa ecología y de ese sistema natural que está en permanente circulación; los cantos, tanto en español como en emberá, evidencian una amalgama de ciertas tecnicalidades ${ }^{25}$ vocales y de signos textuales que despliegan una connotada percepción unívoca por los asistentes. Es decir que, siendo estas prácticas sonoras una marcada herencia colonial campesina, permeada por el incisivo protagonismo de la música «parrandera» ${ }^{26}$ en el territorio, su carácter textual y sus improvisados discursos narrativos, descriptivos y políticos en performance denotan un dispositivo importante en los procesos de construcción de persona, etnia y territorio, delineado en

24 Damaciri es el nombre del grupo musical familiar de don Darío. Es un grupo que resignificó las prácticas musicales campesinas en el territorio y son un referente importante de música indígena en San Lorenzo.

25 Llamo tecnicalidades a las distintas formas ontológicas de aprendizaje dadas en el territorio indígena para con las formas de emisión de la palabra cantada, distinto de los elementos concretos y únicos con respecto a la palabra «técnica» en música.

26 Música «parrandera» alude a un género musical campesino, fiestero y dicharachero - letras satíricas y políticas - difundido por la industria de la música popular contemporánea y adoptada por grupos musicales indígenas en esta zona del país, debido a la movilización intercultural, como lo fue la colonización antioqueña. 
los estados alterados de conciencia ${ }^{27}$ de los participantes, donde se despliegan sentimientos de alegría, llanto y evocación de un pasado y el compromiso afectivo con este, una nostalgia de pensar en algo que se ha tenido o vivido en una etapa anterior y ahora no se tiene o se ha perdido ${ }^{28}$.

Por tanto, las prácticas sonoro-musicales con sus componentes textuales y tímbricos son un aditamento a esos mundos sonoros del entorno ecológico, dimensionado en esa interrrelación de las percepciones sensoriales para otorgar otros beneficios y privilegios en cuanto a la «escucha». Es decir, que el «oler», «palpar», «ver», «saborear»y «escuchar» como la interacción de los sentidos, brindan actos de percepción. Cuando don Darío me dijo «[...] usted por eso debe escuchar muy bien», señala precisamente esa intersensorialidad para otorgar beneficios curativos. Del mismo modo, el taita también hizo mención en esto cuando en el acto de curación-sanación le gritaba al indígena enfermo reiteradamente: « ¡Fredy!, ¡Fredy!, ¡ escuche!, ¡ escuche!, ¡vuelva!», mientras tomaba de la botella el preparado y lo expulsaba en el cuerpo y rostro del indígena.

Las náuseas me generan angustia y sudor frío por no tener mayor control sobre mi cuerpo. Sin embargo, «escucho» todo a mi alrededor, algunos sonidos como los llantos, las risas, los suspiros y los murmullos son el referente auditivo para saber qué está sucediendo en el entorno. Estar de pie o estar sentado me es indiferente, al igual que estar solo o hablar con alguien, al parecer cada uno es testigo solitario de sus angustias y ansiedades. Ahora, con más intensidad segrego saliva en mi boca y con un esfuerzo inmenso empiezo a vomitar unos cúmulos densos que parecen piedras. Algunos autores, relatan que uno de los grandes hechos de los antiguos chamanes consistía en hacer aparecer mágicamente muchas piedras o perlas en el suelo. Don Darío, que está a poca distancia, se aproxima, sin dejar de interpretar su guitarra, y me dice, «no deje de «escuchar» continúe, continúe, pero no deje de escuchar».

27 En varias ocasiones, don Darío me manifestó del éxtasis o estados alterados de consciencia producidos por las performances musicales de Damaciri, tanto en eventos funerales como en fiestas o celebraciones.

28 Siguiendo a Bakhtin (1982) con el concepto de cronotopo, en el cual podemos expresar el carácter indisoluble de espacio y tiempo. Los sonidos de Damaciri representan ese mundo indígena de un territorio anhelado o nostálgico que a la vez se incorpora a ese mundo indígena real-actual enriqueciéndolo, y ese mundo real-actual se incorpora a los textos y al mundo indígena representado en ellos, tanto durante el proceso de composición de esta, como en el posterior proceso de performance musical y el posterior proceso de percepción sonora de los oyentes o receptores indígenas. 


\section{CONCLUSIONES}

Este texto, destaca la multiplicidad de voces que colaboraron en la construcción de interpretaciones en cuanto al sentido del oído (la escucha) (kûrûchobea) indígena. El interés por cómo escuchan los emberá chamí me llevó a pensar y conceder ciertos parámetros metodológicos poco convencionales en la etnografía musical, para aproximar estas elucidaciones en cuanto a la recepción y percepción de todo ese universo sonoro indígena, la cosmosónica emberá chamí.

El sentido del oído (la escucha) (kûrûchobea) para esta sociedad se constituye como parte asidua y fundamental en la construcción del modo de ser/estar indígena. Este trabajo condujo a evidenciar que uno de los caminos para el acceso al saber y conocimiento se consuma a través de las distintas formas de audición (escucha) (kûrûchobea) que establecen y median estas personas indígenas emberá. Por tanto, el análisis semiótico musical de ciertos escenarios sonoro-performáticos fueron comprobados, por la motivación constante de estas personas indígenas para que el investigador fuera participe activo en sus prácticas culturales, con el fin de «comprender» las filosofías y ontologías sonoras.

Esta investigación aporta ciertos conceptos y nociones que derivaron de todo este constructo interpretativo musicológico/etnomusicológico, y que coinciden con un entramado de discusiones con estas identidades indígenas en el campo. Como, por ejemplo, el «percepcionismo sónico» que emerge de la exaltación de la acción de escucha (kûrûchobea) de estas personas indígenas y de la acción de «escucha» del yo-investigador como sujeto que también medió, intervino y percibió en ese complejo sociopolítico-sonoro.

Finalmente, este texto brinda aportes metodológicos en los cuales se dirime, como menciona Faudree (2012), a privilegiar la atención a las dimensiones sonoras por la experiencia social, a través de una etnografía multisensorial. Es decir, la multisensorialidad privilegiada por la escucha o «escucha» amplía posibilidades de aproximación social efectiva mediante todos los sentidos.

\section{REFERENCIAS}

Abu-Lughood, Lila (1991). Writing against culture. En R. Fox, Recapturing anthopology: Working in the present (pp. 137-162). Santafe, New Mexico: School of American Research Press.

Bakhtin, Mijail (1982). Estética de la creación verbal [traducción de Tatiana Bubnova]. México D. F.: Siglo XXI. 
Brabec de Mori, Bernd, Matthias Lewy y Miguel A. García (2015). Sudamérica y sus mundos audibles. Cosmologías y prácticas sonoras de los pueblos indígenas. Berlín: Ibero-Amerikanisches Institut.

Clifford, James y George Marcus (1986). Writing culture: the poetics and politics of ethnography: a school of American Reserach advance seminar. University of California Press.

Erlmann, Veit (2004). Hearing cultures: Essays on sound, listening and modernity. Nueva York: Berg.

Faudree, Paja (2012). Music, Language, and Texts: Sound and Semiotic Ethnography. Annual Review of Anthropology, 41(1), 519-536. https://doi.org/10.1146/ annurev-anthro-092611-145851

Favret-Saada, Jeanne (2005). Ser afetado. Cadernos de Campo (13), 149-153. https:// doi.org/10.11606/issn.2316-9133.v13i13p149-153

Feld, Steven (1982). Sound and sentiment: Birds, Weeping, Poetics, and Song in Kaluli Expression. Filadelfia: University of Pennsylvania.

Feld, Steven (1996). Waterfalls of song: An acoustemology of place resounding in Bosavi, Papua New Guinea. En S. Feld y H. Basso, Senses of place (pp. 91-135). Santa Fe: School of American Research Prees.

García, Miguel (2015). Un oído obediente (y algunas desobediencias). En Bernd Brabec de Mori, Miguel A. García y Matthias Lewy (eds.), Sudamérica y sus mundos audibles: Cosmologías y prácticas sonoras de los pueblos indígenas (pp. 197-211). Berlín: Ibero-Amerikanisches Institut PreuBischer Kulturbesitz.

Howes, David (2010). Sensual relations: Engaging the senses in culture and social theory. Ann Arbor: University of Michigan Press.

Lenkersdorf, Carlos (2008). Aprender a escuchar. México D. F.: Plaza y Valdés.

Lewy, Matthias (2015). Más allá del punto de vista: sonorismo amerindio y entidades de sonido antropomorfas y no-antropoformas. En Bernd Brabec de Mori, Matthiass Lewy y Miguel A. García, Sudamérica y sus mundos audibles. Cosmologías y prácticas sonoras de los pueblos indígenas (pp. 83-98). Berlín: Ibero-Amerikanisches Institut PreuBischer Kulturbesitz.

Menezes Bastos, Rafael José de (1999a). A musicológica kamayurá: para uma antropologia da comunicação no Alto-Xingu. Florianópolis: Editora da Universidade Federal de Santa Catarina.

Menezes Bastos, Rafael José de (1999.b). Apùap world hearing: On the Kamayurá phono-auditory system and the anthropological. The World of Music, 41(1), 85-96. 
Menezes Bastos, Rafael José de (2013). A festa da Jaguatirica, uma partitura críticointerpretativa. Editora Universidade Federal de Santa Catarina.

Nancy, Jean-Luc (2007). A la escucha [traducción de Horacio Pons]. Buenos Aires: Amorrortu.

Ochoa Gautier, Ana María (2015). Aurality: Listening and knowledge in nineteenthcentury Colombia. Durham: Duke University Press.

Oliveiros, Pauline (2005). Deep listening: A composer's sound practice. Lincoln, NE: Universe.

Reichel-Dolmatoff, Gerardo (1960). Notas etnográficas sobre los índios del chocó. Revista Colombiana de Antropología, 9, 73-158.

Santos, Boaventura de Sousa (2009). Una epistemología del sur: la reinvención del conocimeinto y la emancipación social. México: Clacso y Siglo XXI.

Seeger, Anthony (2015). El oido etnográfico. En Bernd Brabec de Mori, Matthiass Lewy y Miguel A. García, Sudamérica y sus mundos audibles. Cosmologías y prácticas sonoras de los pueblos indígenas (pp. 37-36). Berlín: Ibero-Amerikanisches Institut PreuBischer Kulturbesitz.

Seremetakis, C. Nadia (1996). The senses still. Chicago: University of Chicago Press.

Stein, Marilia Raquel Albornoz (2009). Kyringüé in Mborai - os cantos das crianças e a cosmo-sônica Mbyá- Guarani [tesis de doctorado en Etnomusicología/Musicología]. Universidade Federal de Rio Grande do Sul.

Stoller, Paul (1989). The Taste of Ethnographic Thins: The Senses in Anthropology. Filadelfia: University of Pensilvania Press. https://doi.org/10.9783/9780812203141

Taussig, Michael (1993). His Masters Voice. En Mimesis and Alterity; A Particular History of the Senses (pp. 212-236). Nueva York, Londres: Routledge.

Vasco, Luis Guillermo (1975). Los chamí: la situación indígena en Colombia, Colección Minilibros 1. Bogotá: Editorial Margen Izquierdo.

Vasco, Luis Guillermo (1985). Jaibanás: Los verdaderos hombres. Bogotá: Fondo de Promoción de la Cultura del Banco Popular.

Viveiros de Castro, Eduardo (1992). Desde el punto de vista del enemigo: la humanidad y la divinidad en una sociedad amazónica. Chicago: Chicago University Press.

Viveiros de Castro, Eduardo (2002). A inconstância da alma selvagem e outros ensaios de antropologia. São Paulo: Cosac \& Naify.

Voegelin, Salomé (2014). Sonic mundos possible Worlds: Hearing the Continuum of Sound. Nueva York: Bloomsbury. 